\title{
PERCEPCIONES DE PROFESORES Y ESTUDIANTES SOBRE LAS TIC. UN ESTUDIO DE CASO
}

\section{ICT PERCEPTIONS OF TEACHERS AND STUDENTS. A CASE STUDY}

\author{
Ana M. Ortiz Colón; aortiz@ujaen.es \\ Mónica Peñaherrera León; mpleon@ujaen.es \\ Juana M. Ortega Tudela; imortega@ujaen.es \\ Universidad de Jaén
}

\section{RESUMEN}

El trabajo presenta los resultados de un proyecto de investigación desarrollado en el C.E.I.P. Vicente Espinel de Ronda (Málaga), para conocer el impacto de las TIC en dicho Centro, analizándose las percepciones de los profesores a través de la entrevista por un lado y las opiniones de los estudiantes de 5 y 60 de Educación Primaria mediante el cuestionario, por otro. La metodología, aporta conclusiones de actualidad relevantes del uso de las TIC.

PALABRAS CLAVE: Integración tecnológica, uso TIC, educación básica, formación de profesores

\begin{abstract}
The paper presents the results of a research project carried out at the Primary School (C.E.I.P) Vicente Espinel, in Ronda (Málaga) in order to know the impact that ICT has had in this centre. On one hand, the perceptions of teachers are analyzed through the interview and on the other hand, opinions of students in grades 5 and 6 of Primary Education have been taken into account through a questionnaire. The methodology currently provides relevant conclusions from the use of ICT.
\end{abstract}

KEYWORDS: Technology integration, use of ICT, basic education, teacher training. 


\section{INTRODUCCIÓN}

La incorporación de las Tecnologías de la Información y la Comunicación en los Centros Educativos, ha venido a replantear e $\mathrm{n}$ ellos el proyecto educativo, los procesos de enseñanza/aprendizaje y la organización de medios y recursos. Consideramos que los medios son elementos curriculares que intervienen en el proceso de enseñanza en interacción con otros y por tanto la decisión de uso va a depender del proyecto de los centros. A veces el mismo contexto físico, curricular, cultural, facilita o dificulta si el medio debe ser utilizado o no, siguiendo la línea planteada por Cabero (1998). Son muchos los informes e investigaciones acerca de la integración de las TIC en el ámbito educativo (Area, 2005; Balanskat A.; BECTA (2006); Cabero, 2001; Cattagni y Farris, 2001; Cebrián, Ruíz y Sánchez, 2008; Cebrián, Ruiz y Rodríguez, 2007; Fandos, 2007; Marchesi y Martin, 2003; Pérez y Sola, 2006). En ellos se pone de relieve los crecientes esfuerzos de las administraciones para la dotación y adaptación tecnológica de los centros educativos.

Desde una perspectiva diacrónica, las investigaciones sobre medios de enseñanza en los últimos años han respondido fundamentalmente a tres corrientes epistemológicas: tecnológico/positivista; simbólico/interactiva y curricular (Area, 1991). Este perfil permite identificar la evolución del papel de los medios y sus posibles aplicaciones en los procesos de aprendizaje; las investigaciones sobre los dos primeros enfoques utilizaron preferentemente metodologías de base cuantitativa; si bien la perspectiva curricular, se ha apoyado en metodologías originadas en el ámbito de los estudios de pensamiento y la acción profesional de los profesores, siendo la figura docente el primer factor de innovación. Los medios, desde esta perspectiva han sido estudiados con metodologías descriptivas, desarrollando aspectos referidos al conocimiento de las concepciones sobre los profesores, el conocimiento de los contextos de uso, entre otros.

En nuestro país destacan numerosas investigaciones y estudios sobre las TIC en la educación, la mayoría de corte cuantitativo. En cambio, son escasas las investigaciones en profundidad de enfoque cualitativo, sobre las repercusiones organizativas, profesionales y curriculares de su introducción en la educación. Area (2005) hace una revisión de las líneas de investigación en TIC en el sistema educativo. Una de las líneas denominada estudios sobre los usos y prácticas pedagógicas con ordenadores en contextos reales de centros y aulas, indica que se trata de estudios con planteamientos metodológicos cualitativos de estudios de caso (Ortiz, 2006).

Por otro lado, Montero, Gewerc, Vidal, Fernández, Rodríguez, González Guisande, González Fernández, Pernas y Martínez (2004) estudian la influencia de las TIC en el desarrollo organizativo y profesional de los centros educativos en Galicia, con dos fases, una cuantitativa y otra cualitativa con estudios de caso. En el contexto andaluz, Boza, Toscano y Méndez (2009) analizan asimismo, el impacto de los proyectos TIC andaluces en la organización y los procesos de enseñanza-aprendizaje desde un enfoque investigador de carácter mixto. Por otro lado, destacan los estudios realizados por Valverde, Garrido y Sosa, (2010) y Boza, Tirado y Guzmán (2010), en referencia a las dificultades e integración del uso de las TIC en la enseñanza.

\section{METODOLOGÍA DEL ESTUDIO}

En nuestro estudio se planteó la necesidad de conocer en profundidad cómo se estaba 
desarrollando la incorporación de las TIC en el centro educativo, analizando en profundidad las percepciones de los agentes implicados. Para ello se optó por una metodología mixta que combinó el método de encuesta para describir las opiniones de los estudiantes en su acercamiento a las TIC y la entrevista a los profesores del centro, los cuales nos aportaron información sobre las dificultades y problemas en el Estudio de Caso diseñado, a través de la complementariedad de técnicas e instrumentos creados al efecto.

\subsection{Objetivos}

El objetivo general es describir y analizar en profundidad el uso de las TIC en el Contexto del Centro Educativo, desde la perspectiva de los estudiantes y profesores.

Este objetivo, se concreta en los siguientes objetivos específicos:

1. Valorar los procesos de interacción a través de las TIC que desarrolla el profesorado en el contexto del Centro educativo.

2. Indagar sobre el uso de las TIC que realiza el alumnado en los procesos de enseñanza/aprendizaje.

3. Describir y analizar, las necesidades formativas del profesorado en la Sociedad de la información.

4. Obtener y mostrar información sobre el uso e integración de las TIC en la mejora del centro en su conjunto.

\subsection{Estrategias metodológicas}

Para obtener una visión general de la situación se ha recurrido a la utilización de diversas estrategias metodológicas con la intención de triangular los resultados, obteniendo así un mayor nivel de validez de las conclusiones.

El diseño del estudio, incluye una cuestionario dirigido al alumnado de 5o y 60 de Educación Primaria que ha tratado de explorar el uso que el alumnado hace de las TIC. La validación del instrumento se realizó a través del juicio de expertos (cinco profesores universitarios y cinco profesionales), quedando estructurada la versión definitiva en tres dimensiones que versan sobre: "el equipamiento familiar en tecnologías de la información y comunicación" (corresponde a los ítems que van del número 1 al 12), "la formación en tecnologías de la información y comunicación" (abarca los ítems número 13 al 24), y "la interacción de las TIC en el ámbito escolar" (corresponde con los ítems número 25 al 32). El cálculo de la fiabilidad se realizó a través del coeficiente de correlación alfa de Cronbach, obteniendo un valor de 0,8827 , utilizando el programa Reliability en SPSS 
La segunda estrategia, la entrevista, pretende obtener la visión de la realidad de los profesores con relación a las TIC en el centro y aula. Estas dos fuentes de información permiten una contrastación de las distintas conclusiones, enriqueciendo los resultados del proyecto y sus aplicaciones futuras a la realidad educativa de Andalucía en particular y de los centros educativos en general.

\subsection{Contexto y participantes del estudio}

Los participantes en el estudio han sido profesores y estudiantes del Centro Vicente Espinel de Ronda (Málaga). Desde la dirección del Centro educativo se realizaron las gestiones oportunas para acceder a las distintas fuentes de información (alumnado y profesorado). Para ello, se informó de los objetivos del estudio, y se solicitó la participación en el mismo de forma voluntaria junto con los consentimientos informados de tutores/as, o padres y madres.

Han participado cuarenta y un estudiantes pertenecientes a los cursos de 50 y 60 de Educación Primaria, a los cuales se accedió por ser el segmento de discentes que cuenta con equipamientos informáticos en el aula, así como un diseño curricular que contempla la utilización integrada de los mismos. Los cuestionarios se administraron en horas de clase, utilizando para ello parte del horario de las mismas. El tiempo de realización no superó los treinta minutos.

Los profesores entrevistados fueron docentes voluntarios. Del grupo total, solamente participaron tres por razones de horario laboral y compromisos personales. En el caso de los profesores, las entrevistas fueron realizadas por personal de apoyo especializado en la realización de entrevistas personales.

\begin{tabular}{|l|l|}
\hline $\begin{array}{l}\text { Fuentes de información } \\
\text { (participantes) }\end{array}$ & Número \\
\hline Profesores y profesoras & 3 \\
\hline Estudiantes & 41 \\
\hline
\end{tabular}

Tabla 1. Participantes en el estudio

Las entrevistas, realizadas a finales de noviembre de 2010, tuvieron una duración mínima de treinta minutos y máxima de cuarenta minutos. Todas las entrevistas se realizaron en aulas del colegio situadas en la primera planta del mismo, utilizando una grabadora digital de audio, que permitió la posterior transcripción de las entrevistas, facilitando así su análisis. El procedimiento seguido incluía las siguientes fases:

- Definición de la estructura de categorías. Para ello se siguió un procedimiento emergente, de forma que a partir de la lectura de las transcripciones, procedentes de las grabaciones de las entrevistas, se identificaron distintos códigos que permitieron la codificación del texto, así como la relación entre ellos (estructura).

- Organización de las categorías, para lo cual se utilizó una matriz textual.

- Extracción de conclusiones a partir de dos procedimientos, por un lado la triangulación de analistas (comparando las conclusiones que cada analista había extraído de forma independiente) y por otro la saturación (revisión sistemática, crítica y reiterativa de las conclusiones, del texto y de la codificación, tratando 
de identificar resultados a favor y en contra de dichas conclusiones).

\section{RESULTADOS}

\subsection{Cuestionario}

Los resultados recogidos en el cuestionario dirigido al alumnado de 50 y 60 de Educación Primaria, sobre el estudio y análisis del uso de las TIC en el contexto del Centro Vicente Espinel, se estructuraron en torno a las tres categorías establecidas en el diseño del instrumento. De las 41 personas que participaron en la valoración de los cuestionarios, 17 fueron alumnos y 24 alumnas. Dicha muestra correspondió a 19 estudiantes pertenecientes a 50 curso de Primaria y 22 a 60 curso de Primaria, con edades que comprendieron entre los 10 y los 12 años (en concreto 14 estudiantes de 10 años, 18 estudiantes de 11 años y 9 estudiantes de 12 años).

\section{Equipamiento familiar en TIC (ítem 1 al 12)}

Tal como ya se ha indicado, los primeros ítems del cuestionario aportan información sobre el "equipamiento familiar en relación con las tecnologías de la información y comunicación".

Si bien es cierto que todo el alumnado participante manifiesta tener televisor en su casa, en el resto de los ítems pertenecientes a esta categoría se observan diferencias (tabla 1), donde se destaca lo siguiente: una gran mayoría tienen o disponen de: "ordenador de mesa" (32 personas frente a los 8 que dicen no tenerlo), "otro tipo de tecnologías, del tipo de la Play Station, la WII o la PDI" (36 estudiantes dicen poseerlo frente a los 5 que lo niegan), "teléfono móvil" (38 que sí frente a 3 que no). Siendo inferiores las diferencias en el resto de los ítems.

\begin{tabular}{|l|l|l|l|}
\hline \multicolumn{2}{|l|}{ Tabla . Frecuencia de los ítems 1 al 11 } & SI & NO \\
\hline No & Ítem. Posee o dispone en casa de: & 41 & 0 \\
\hline 1 & Televisión & 32 & 8 \\
\hline 2 & PC u ordenador de mesa & 17 & 24 \\
\hline 3 & Ordenador portátil & 36 & 5 \\
\hline 4 & $\begin{array}{l}\text { Otro tipo de NNTT: Play Station, WII, PDA, Agenda electrónica } \\
\text { Nintendo,... }\end{array}$ & 37 & 4 \\
\hline 5 & Teléfono fijo & 38 & 3 \\
\hline 6 & Teléfono móvil & 35 & 6 \\
\hline 7 & Radio, radiocassette o transistor & 21 & 17 \\
\hline 8 & Cadena musical, equipo de alta fidelidad o laserdisk & 33 & 8 \\
\hline 9 & Vídeo & 35 & 6 \\
\hline 10 & DVD & 26 & 15 \\
\hline 11 & Conexión internet & & \\
\hline
\end{tabular}

Tabla 2. Frecuencia de los ítems 1 al 11

Por último, hay un ítem que no está recogido en la tabla pero que se encuentra dentro de este punto: "la posesión de correo electrónico". De los 39 estudiantes que han respondido, la mayoría tienen correo electrónico y "sí" lo utilizan a menudo (17 estudiantes). Por el contrario, 13 dicen "no" tener correo electrónico, seguido de los 9 estudiantes que dicen "tenerlo pero que no lo utilizan a menudo". 
Formación del alumnado en tecnologías de la información y comunicación (ítem 13 al 24)

En la vida diaria, el alumnado hace distintos usos del ordenador, donde la principal función que le asignan es la de "jugar" (26 estudiantes), seguida de la realización de "trabajos en clase" (24 personas) y de la "navegación por internet" (19 estudiantes) (ver ilustración 1).

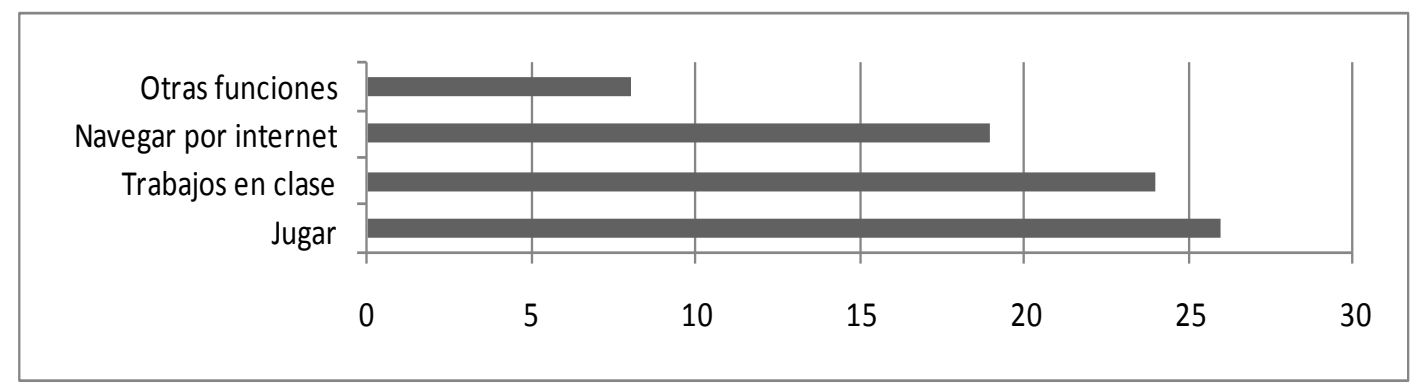

Ilustración 1. Usos que hacen los estudiantes del ordenador

Centrando las preguntas en el uso que el alumnado hace de internet, la opción mayoritaria es el "Messenger" (22 estudiantes que lo utilizan frente a los 19 que no), seguida de la "descarga de archivos, como son las películas o la música" (18 que reconocen darle esa función a internet), "realización de búsquedas" (9 son los estudiantes que utilizan los buscadores dentro de la red) y de uso del "correo electrónico" (5 estudiantes que dan este uso). (Ilustración 2).

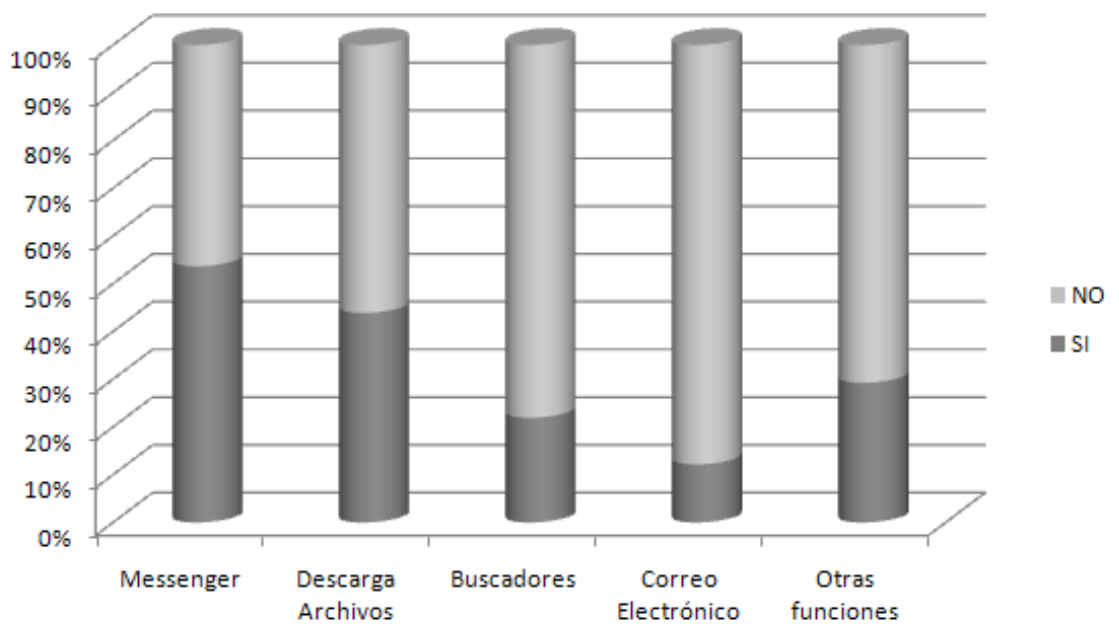

Ilustración 2. ¿Para qué utilizas internet?

En relación al conocimiento y habilidades que el alumnado manifiesta tener sobre el manejo del ordenador, 27 han respondido que "saben cambiar la resolución de la pantalla del monitor", 37 dicen que "saben crear una carpeta" y 29 afirman "conocer cómo copiar documentos".

\begin{tabular}{|l|l|l|l|}
\hline \multicolumn{2}{|l|}{ Tabla 3. Frecuencia de los ítems 15, 16, 17, 18, 19, 20, 25, 26, 30, 31 y 32 } \\
\hline № & Ítem & $\mathrm{SI}$ & $\mathrm{NO}$ \\
\hline 15 & Sabe cambiar de resolución la pantalla del monitor & 27 & 12 \\
\hline 16 & Sabe crear una carpeta & 37 & 4 \\
\hline 17 & Sabe copiar y pegar documentos & 29 & 12 \\
\hline
\end{tabular}




\begin{tabular}{|c|c|c|c|}
\hline 18 & Sabe cómo grabar un CD de datos & 24 & 16 \\
\hline 19 & $\begin{array}{l}\text { Sabe conectar periféricos al PC: equipos de audio, cámara } \\
\text { digital,... }\end{array}$ & 23 & 17 \\
\hline 20 & Sabe adjuntar archivos a un correo electrónico & 27 & 13 \\
\hline 25 & Utiliza el ordenador en el colegio & 40 & 1 \\
\hline 26 & $\begin{array}{l}\text { Cree que necesita mejorar el manejo del ordenador para } \\
\text { utilizarlo en clase }\end{array}$ & 29 & 7 \\
\hline 30 & $\begin{array}{l}\text { Utiliza en clase programas informáticos de apoyo, como: JClic, } \\
\text { Adibu, Pipo,... }\end{array}$ & 26 & 11 \\
\hline 31 & Recibe información sobre el colegio a través de internet & 16 & 21 \\
\hline 32 & $\begin{array}{l}\text { Las reuniones de tutoría se convocan por medio de internet, } \\
\text { web, e-mail,... }\end{array}$ & 16 & 21 \\
\hline
\end{tabular}

Tabla 3. Frecuencia de los ítems 15, 16, 17, 18, 19, 20, 25, 26, 30, 31 y 32

La interacción de las TIC en el ámbito escolar (ítem 25 al 32)

En esta última categoría se ha podido saber que todos los estudiantes "utilizan el ordenador en el colegio", de los cuales, sólo 6 reconocieron que "necesitaban mejorar el manejo del ordenador para utilizarlo en clase" frente a los 31 que valoraron no necesitarlo (Tabla 3).

Al consultar el tiempo que utiliza el ordenador diariamente el alumnado dentro del horario de clase, la mayoría elige la opción "menos de 1 hora" (29 respuestas) seguida de "de 1 a 3 horas" (con 7 respuestas).

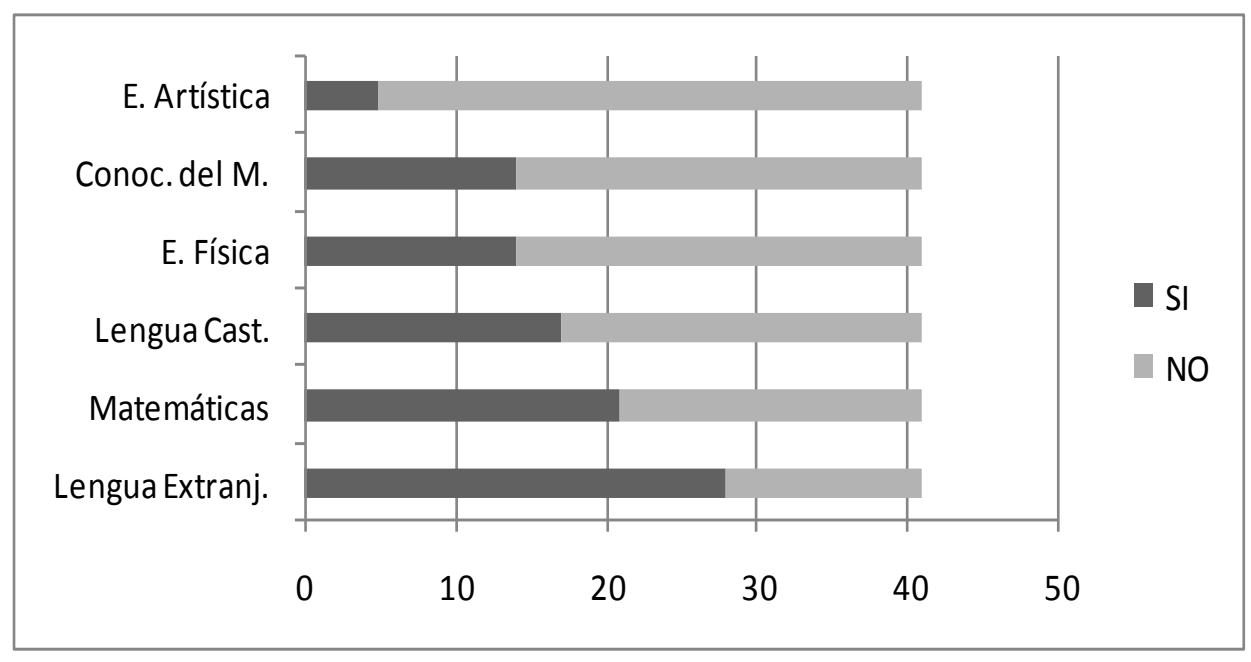

Ilustración 3. Asignaturas en las que se utiliza el ordenador

De manera más concreta, al encuestar al alumnado con relación a las asignaturas donde se utiliza el ordenador, la materia en la cual más se utiliza es "lengua extranjera" (28 estudiantes) frente a los 13 que dicen no hacerlo, seguida de "matemáticas" (21 estudiantes). (Tabla 2 e ilustración 3 ). 


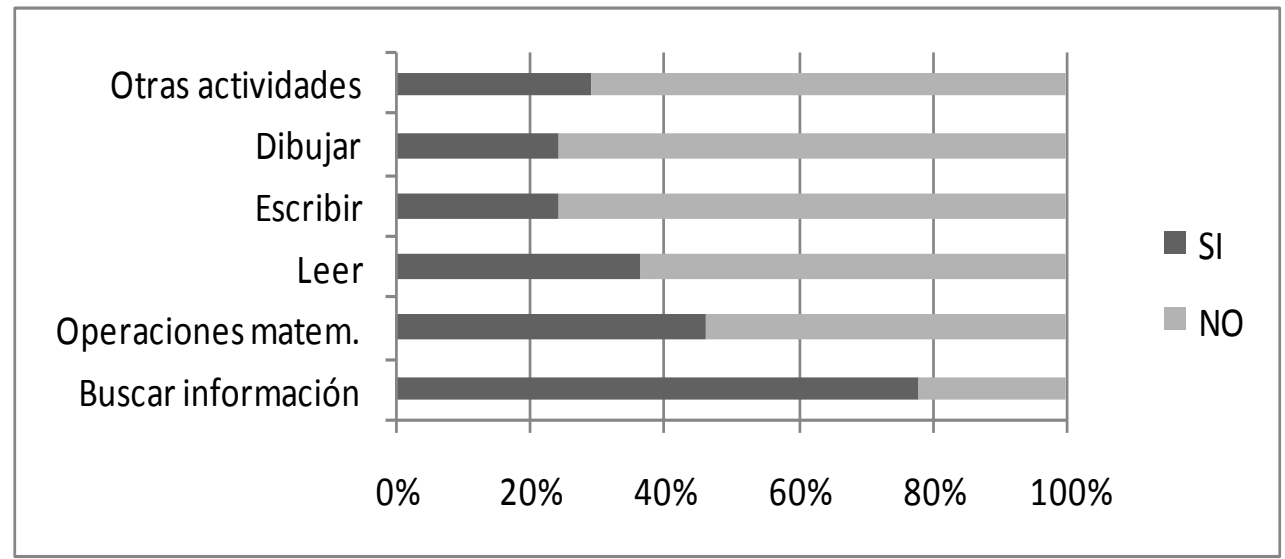

Ilustración 4. Actividades que se realizan en clase con el ordenador

Así mismo, se les ha preguntado si "utilizan en clase programas informáticos que les sirva de apoyo" dentro de las mismas (como son el JClic, el Adibu o el Pipo), donde el $14,6 \%$ del alumnado participante reconoce que sí (tabla 3).

Por último, en relación al uso comunicativo y de información que se hace de las TIC desde el centro en relación con el alumnado, se les ha consultado si:

- "A través de internet reciben información del centro", a lo cual 16 estudiantes afirman que sí frente a los 35 que dicen que no.

- "A través de internet (web, e-mail, ... ) se convocan las reuniones de tutorías", a lo cual 6 han respondido afirmativamente ante los 31 que no lo saben.

\subsection{Entrevista a profesores}

Se realizaron tres entrevistas a otros tantos docentes, dos profesoras y un profesor. Estas entrevistas se efectuaron dentro de un ambiente distendido donde los docentes dialogaron, dentro de un formato de entrevista semiestructurada, con el entrevistador. El proceso de análisis de las entrevistas, se realizó utilizando el programa informático de análisis cualitativo Atlas.ti 6.0, para darle un mayor rigor a las conclusiones obtenidas y ser de gran ayuda en la utilización de los datos cualitativos. Tal y como se indicó anteriormente, se organizó en tres fases: definición de la estructura de las categorías, organización de las categorías y extracción de conclusiones.

\subsubsection{Categorización: estructura de códigos}

Tras la lectura de la transcripciones correspondientes a las entrevistas realizadas a los docentes, se identificaron 8 categorías básicas y subcategorías: USO, UPR, ACTITUD, PERSPECTIVAS, INT, FORMACIÓN, DEFICIENCIAS, OTROS.

\subsubsection{Organización de las categorías informativas y extracción de conclusiones}

La codificación de las entrevistas ha permitido extraer las primeras conclusiones sobre la realidad de las TIC en el centro escolar. Para una mejor visualización de la información aportada, se ha construido una matriz textual, organizada por entrevista y por categorías, cuyo resumen se incluye a continuación (tabla 3). 


\begin{tabular}{|c|c|c|c|}
\hline & ENTREVISTA 1 & ENTREVISTA 2 & ENTREVISTA 3 \\
\hline \multirow[t]{2}{*}{ ACTITUD_+ } & [3357: 3824] & & \\
\hline & [ 2554: 2715] [ 2758: 3339] [ 3357: [ & [ 9059: 9397] [12567:13242] & \\
\hline ACTITUD_- & 3734] [ 5877: 6048] & $\begin{array}{l}{[13245: 13434]} \\
{[\text { 6685: 6901] [ 7490: 8020] }}\end{array}$ & \\
\hline DEF & [ 9314: 9687] & [11956:12534] [14203:14671] & [16093:16455] \\
\hline \multirow[t]{2}{*}{ FORMA_C } & [ 1371: 2715] & [ 1370: 1560] [ 3715: 5380] & [11058:12189] \\
\hline & & [2607:2946] [5542:6063] & \\
\hline FORMA_D & & [6902:7437] & \\
\hline \multirow[t]{3}{*}{ INT_ACTITUD_+ } & {$[6935: 7501]$} & & [3205:3918] \\
\hline & & & [13537:13990] \\
\hline & & & [14411:14907] \\
\hline INT_DIFICUL & & [ 8069: 8570] [ 9406:10425] & {$[16457: 17282]$} \\
\hline INT_DIF_ALU & & & [14923:15587] \\
\hline OTROS & [1101:1347] [9314:9687] & & \\
\hline PERSPECTIVAS_+ & & [10976:11249] & [15588:15875] \\
\hline PERSPECTIVAS_- & [8903:9187] & & \\
\hline UPR_C & {$[19: 270]$} & & \\
\hline UPR_DOC & {$[19: 270]$} & & \\
\hline \multirow[t]{2}{*}{ UPR_E } & [116:943] & [ 31:142] [262:488] & [ 28:462] \\
\hline & & & {$[1385: 1634]$} \\
\hline UPR_I & [ 815: 944] & & [1923:2022] \\
\hline UPR_OF & & [3248:3590] & [3087:3376] \\
\hline UPR_OT & & & [544:2417] \\
\hline USO_+ & [3267:4978] [8926:9187] & [1667:1961] & [3205:3918] \\
\hline \multirow[t]{2}{*}{ USO_- } & {$[3735: 5231][6935: 7501]$} & [2015:2496] [6685:6901] & \\
\hline & & [ 979: 1310] [ 1667: 1961] & \\
\hline USO_P & & [13245:13434] & \\
\hline
\end{tabular}

En el siguiente cuadro (tabla 5) se recoge el número de codificaciones para cada código, donde se puede observar que las categorías, actitud negativa, dificultades de interacción en el aula y deficiencias son las que más destacan, mientras que las categorías actitud negativa del alumnado en el aula (INT_ACTITUD_ALU_-), uso elevado del equipamiento TIC en el aula (USO_M), y utilización del equipamiento personal de cada docente (los equipos de casa) (UPR_F) no tienen ningún registro.

\begin{tabular}{|l|l|l|l|l|}
\hline ACTITUD_+ & ACTITUD_- & DEF & FORMA_C & FORMA_D \\
\hline 1 & 7 & 6 & 4 & 3 \\
\hline INT_ACTITUD_ALU_+ & INT_DIFICUL & INT_DIF_ALU & OTROS & PERSPECTIVAS_+ \\
\hline 2 & 5 & 1 & 2 & 2 \\
\hline PERSPECTIVAS_- & UPR_C & UPR_DOC & UPR_E & UPR_I \\
\hline 1 & 1 & 1 & 4 & 3 \\
\hline UPR_OF & UPR_OT & USO_+ & USO_- & USO_P \\
\hline 2 & 1 & 4 & 4 & 3 \\
\hline
\end{tabular}

\subsubsection{Resultados de las entrevistas}

La revisión de la estructura categorial y la codificación de las entrevistas aportan algunas líneas interesantes que podemos considerar como conclusiones. Así, solamente un entrevistado manifiesta una actitud positiva a priori ante la digitalización del aula (segmento 01_Entr[3357:3824]), mientras que la actitud negativa está ampliamente presente en dos entrevistados, con afirmaciones como: 
Entrevista 1: Que eso te tiene que gustar o necesitarlo para el trabajo y como no lo necesito y no me gusta.

Entrevista 2: Pero tampoco lo veo imprescindible. Lo veo mas por lo de los libros que en vez de hacerlo en papel...y que con el tiempo sale mas barato.

En este mismo sentido, existen quejas generalizadas sobre problemas técnicos y falta de apoyo logístico con los equipamientos (segmento 02_Entr[14203:14671]) poniendo de manifiesto serias deficiencias en el seguimiento de las iniciativas TIC. Por su parte, los entrevistados manifiestan haber realizado diversos cursos sobre TIC, sin embargo estos suelen ser poco actuales. Los docentes ponen de manifiesto no solamente su falta de formación, sino también el hecho de verse superados por la capacidad del alumnado a la hora de aprender a utilizar los ordenadores:

Pues ya está hemos llegado tarde. Y ahora cualquier niño es un lince.

Se mete en todos los lados pero nosotros no y como esto cambia

Esto de un día para otro.

Dentro del aula, parece que existen serios problemas para la dinámica de las clases cuando los equipos no se utilizan como un recurso integrado en el proceso de enseñanza y aprendizaje. Se llega a afirmar que los maestros no pueden ver lo que hacen porque las pantallas se lo impiden.

_ Y eso ¿Qué acabas de decir...Que no es demasiado útil?

_No lo veo necesario los niños aprenden a manejar el ordenador sin que lo tengan al lado

_ Sin que lo tengan al lado

_Y da lo mismo que lo tenga aquí en clase

Esto en principio es más incomodo que otra cosa, nos tapan, están juntos, no hemos avanzado

A partir de estos resultados, junto con la revisión de la categorización, se propone el siguiente esquema sobre cómo se relacionan entre sí los distintos factores comentados (gráfico 1). La información recogida pone de manifiesto una serie de deficiencias que afectan a los profesores y a su actividad diaria. En primer lugar los problemas técnicos de los equipos informáticos y la falta de un servicio técnico rápido y eficaz. A esto se une la falta de un conocimiento generalizado sobre el software disponible, o incluso la ausencia de aplicaciones útiles para las distintas materias. Otro factor importante es la deficiencia de formación de los docentes en cómo utilizar eficazmente (de forma integrada) las tecnologías en la enseñanza. Esto puede estar afectando a la actitud que manifiestan los profesores con relación a las TIC en la realidad docente actual. Todo ello puede estar fomentando y alimentando un efecto recursivo que favorece el uso deficitario de las TIC en el aula. No obstante, existe una idea positiva con relación al futuro de la tecnología. 


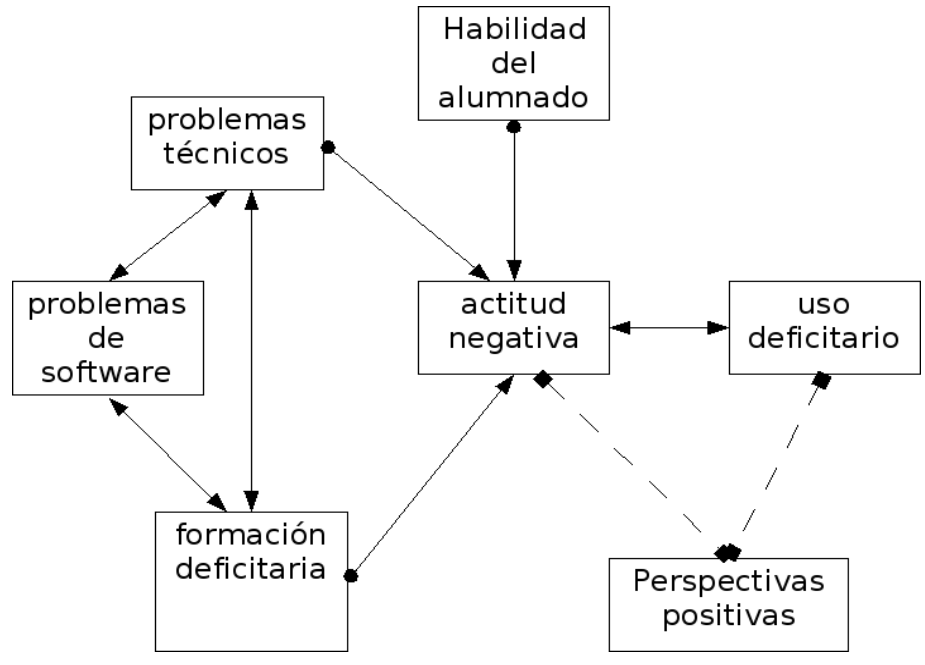

Gráfico 1. Mapa de relaciones entre factores

\section{CONCLUSIONES FINALES}

Las conclusiones se organizan en torno a los objetivos de la investigación y los resultados obtenidos en otras investigaciones referidas a las perspectivas de los agentes educativos hacia las TIC y el uso en contextos escolares, centradas éstas en el papel del profesorado y el alumnado, las TIC en el Aula y en el Centro, y los aspectos positivos y negativos hallados.

A nivel de centro, en general, se ha producido una mayor colaboración y comunicación entre los profesores, así como un incremento de la implicación del profesorado en las actividades de formación. También hemos detectado, y coincidimos con Pérez Gómez y Sola (2006), que se incrementan las actividades de intercambio y coordinación del profesorado al compartir recursos, información, archivos, enlaces, y estrategias metodológicas en el aula.

El proyecto de integración de las tecnologías de la información en el ámbito educativo tiene un nivel considerable de integración didáctica en las aulas del centro, al estar asumido por el equipo directivo, el apoyo de la Administración Educativa (Proyectos de Innovación educativa y Proyectos de Investigación de la Junta de Andalucía) y del claustro de profesores y Consejo escolar. Por su parte, Pérez, et al. (2010) en la línea de la implantación masiva de las TIC, destacan la necesidad de consolidar la profesionalización de los equipos directivos y la figura del coordinador TIC como dinamizadores del proceso de innovación, cambio de mentalidad del profesorado, y la necesidad de renovación de los modelos didácticos en el profesorado para el cambio metodológico en el proceso de enseñanza/aprendizaje, lo que viene a confirmar la conclusión a la que llegamos en nuestro trabajo de la importancia del apoyo institucional en la incorporación de las TIC en el centro y aula del estudio.

Se observa el impacto de la incorporación de las TIC en la práctica en pequeñas innovaciones educativas de los profesores en su metodología en el aula en referencia fundamentalmente a la información, siendo más escasa en procesos de comunicación, en línea con las afirmaciones de Cebrián et al. (2008), del nuevo papel de los estudiantes en clase. 
El uso de estos nuevos recursos ha supuesto una mayor motivación e implicación de los estudiantes en la clase, no exenta de procesos de ansiedad en determinados estudiantes que disponen de menor competencia tecnológica. En este caso se observan diferencias relevantes en el uso de programas JClic, Adibu, Pipo, a favor de los alumnos $(54,1 \%)$ respecto a las alumnas $(29,7 \%)$. Destaca por otro lado un mayor tiempo de uso del ordenador diariamente en el aula en referencia a la edad. Se observa la relación existente entre algunas variables destacando como los más significativos, los contextos familiares que gozan de mayores recursos tecnológicos (95\%), asociados al disfrute de conexión a internet y correo electrónico en el hogar (96\%), coincidiendo con los resultados planteados por Cebrián et al. (2008), en la influencia del Proyecto TIC en los hábitos de los estudiantes cuando manejan el ordenador en casa. El uso que hacen de internet responde en orden descendente al Messenger, descargas, buscadores y correo electrónico como los más destacados.

La mayoría de los profesores declaran utilizar las TIC regularmente en sus clases, mientras que algo más de una tercera parte afirma no usarlas con tal regularidad, coincidiendo estas conclusiones con los datos aportados por Boza et al. (2010), sobre la inserción de las TIC en los centros educativos andaluces y las creencias del profesorado sobre la integración de las TIC y la formación que han recibido sobre las mismas. Por tanto, los resultados confirman que los docentes hacen una mayor integración y uso a medida que su nivel de conocimientos en recursos tecnológicos aumenta, ajustándose a los hallados en la literatura (Área, 2005; Almerich et al., 2005).

Las asignaturas en las que más utilizan las TIC son: lengua extranjera (68\%), matemáticas (52\%), lengua castellana y literatura, educación física, conocimiento del medio, social y cultural y educación artística. Las TIC se utilizan, fundamentalmente, para buscar información y reforzar los aprendizajes, traductores, diccionarios, entre otros.

Para el profesorado tanto los centros como los profesores innovadores no son fruto de la integración de las TIC, sino que ambos lo eran antes de la llegada de las TIC; las TIC le han permitido potenciar su innovación mediante herramientas que posibilitan nuevas acciones $u$ optimizar las que realizaban antes, pero su modelo educativo no ha cambiado sustantivamente, siendo coincidentes los resultados con los de Almerich et al. (2010).

El profesorado entrevistado, se muestra dudoso, pero optimista, con los avances alcanzados, entendiendo por otro lado que se han dado algunos pasos importantes y que aún queda mucho por hacer. En esta línea encontramos coincidencias en los resultados mostrados por Boza et al. (2009), al considerar que los efectos de las TIC no deben tenerse en cuenta hasta que no se produzca una verdadera integración en la dinámica del aula. Por su parte Cebrián et al. (2008), coinciden en expresar la falta de reflexión durante la práctica en la implementación de las TIC, confirmándose los avances planteados por el profesorado en la investigación.

Muestran expresamente la necesidad de formación especializada en TIC en referencia a aplicaciones útiles y específicas para las distintas materias. De este modo, encontramos claras coincidencias con las relaciones entre las creencias del profesorado, en relación a la integración de las TIC y la formación que han recibido sobre ellas (Boza et al. (2010).

En líneas generales el profesorado considera un éxito las medidas de impulso de la Junta de Andalucía para acercar al alumnado a la sociedad del conocimiento, a través de los programas de Centros TIC promovidos en el año 2003. Lo deseable es la integración de las TIC con normalidad en la vida del centro. Puede afirmarse tal éxito, en la medida que se 
están produciendo proyectos innovadores que implican una mejora del rendimiento académico de los estudiantes de Educación Primaria en el contexto del centro. Estos efectos positivos, son claves, de cara a la motivación e interés del alumnado, coincidiendo con las aportaciones de Boza et al. (2009) y Pérez, et al. (2010).

Se ha producido por otro lado una mejora respecto a la dotación de equipamiento y mobiliario, gracias a una política flexible que les ha permitido disponer de equipos y materiales requeridos por el centro y adaptados a las necesidades planteadas en el Proyecto TIC y sucesivas convocatorias de apoyo al proyecto inicial. Se puede afirmar que la integración de las TIC en el centro ha contribuido en gran medida a la mejora del centro en su conjunto.

Entre los aspectos negativos, se considera la inestabilidad de la plantilla como uno de los factores que condiciona la integración de las TIC en el progreso del Centro. Por otro lado los problemas técnicos y de coordinación han sido dificultades a lo largo del proceso de incorporación de las TIC, así como la comunicación con las familias (Cebrián et al., 2008), que no ha alcanzado los objetivos deseados hasta la fecha, coincidiendo con las dificultades encontradas por Boza et al. (2010) en referencia a obstáculos en la integración de las TIC, tanto externos (técnicos, tiempos, apoyos), como internos (actitudes, creencias, prácticas y resistencias), que influyen en estos procesos, así como los planteados por Valverde et al. (2010).

Otro aspecto a destacar como mejorable, es la necesidad de formación en TIC de forma integrada en el currículum junto al conocimiento de buenas prácticas, conclusión que se confirma con el trabajo desarrollado por Boza y Toscano, (2011) en relación a buenas prácticas en integración de las TIC en educación en Andalucía, en referencia a la necesidad de un modelo didáctico en la aplicación de las TIC, fundamentalmente y las recomendaciones expresadas en su momento por Cabero, (2003) en referencia a la adopción de medidas para la formación del profesorado, y para la realización de proyectos conjuntos de interacción a través de las tecnologías.

\section{REFERENCIAS BIBLIOGRÁFICAS}

Almerich, G., Suárez, J. M., Orellana, N. y Díaz, M.I. (2010). La relación entre la integración de las tecnologías de la información y comunicación y su conocimiento. Revista de Investigación Educativa. 28 (1), 31-50.

Almerich, G., Suárez, J., Orellana, N., Belloch C., Bo, R. y Gastaldo, I . (2005). Diferencias en los conocimientos de los recursos tecnológicos en profesores a partir del género, edad y tipo de centro. Revista Electrónica de Investigación y Evaluación Educativa. 11(2), 127-146.

Area, M. (2010). El proceso de integración y uso pedagógico de las TIC en los centros educativos. Un estudio de casos. Revista de Educación, 352; 77-97.

Area, M. (2005). Tecnologías de la información y comunicación en el sistema escolar. Una revisión de las líneas de investigación. RELIEVE: v. 11, n. 1, p. 3-25. Disponible en: http://www.uv.es/RELIEVE/v11n1/RELIEVEv11n1 1.htm 
Area, M. (1991). Los medios, los profesores y el currículo. Barcelona, Sendai.

Balanskat A.; Blamire R. et al (2006). The ICT Impact Report, Download at: http://insight.eun.org/shared/data/pdf/impact study.pdf

BECTA (2006). The Becta Review 2006: Evidence on the progress of ICT in education, UK, Becta.

Boza, A. y Toscano, M.O. (2011). Buenas prácticas de la integración de las TIC en educación. Ponencia VI Congreso Virtual de AIDIPE. Disponible en: http://www.uv.es/aidipe/congresos/Ponencia VIICongresoVirtual AIDIPE.pdf

Boza, A., Tirado, R. y Guzmán M. (2010). Creencias del profesorado sobre el significado de la tecnología en la enseñanza: influencia para su inserción en los centros docentes andaluces. Revista Relieve, 16(1), 1-24.

Boza, A., Toscano, M.O. y Méndez, J.M. (2009). El impacto de los proyectos TIC en la organización y los procesos de enseñanza-aprendizaje en los centros educativos. Revista de Investigación Educativa, vol. 29, no 1; 263- $289 . \quad$ Disponible en http://revistas.um.es/rie/article/view/94401/103031

Cabero, J. (Coord.) (2007). Nuevas tecnologías aplicadas a la educación. Madrid: McGrawHill.

Cabero, J. y Llorente, C. (Dirs.) (2006). La Rosa de los Vientos, Dominios tecnológicos de las TIC por los estudiantes. Sevilla: GID.

Cabero, J. (2003). Las andaduras de Andalucía en las TICs aplicadas a la enseñanza. En XXI Revista de Educación, 5, 27-50.

Cabero, J. (2001). Tecnología educativa. Diseño y utilización de medios de enseñanza. Barcelona: Paidós.

CABERO, J. (1998): Impacto de las nuevas tecnologías de la información y la comunicación en las organizaciones educativas, en LORENZO, M. y otros (coords): Enfoques en la organización y dirección de instituciones educativas formales y no formales, Granada, Grupo Editorial Universitario, 197-206.

Cattagni, A. y Farris, E. (2001). Internet Access in US. Public Schools and Classrooms: (19942000). National Center for Education Statistics.

Cebrián, M. y Ruíz, J. y Sánchez, J. (2008). Impacto producido por el proyecto de centros TIC en CEIP e IES de Andalucía desde la opinion de docents. Revista Pixel-Bit, 31, 141-154.

Cebrian, M. Ruíz, J. y Rodríguez, J. (2007). Estudio del impacto del Proyecto TIC desde la

Opinión de los docentes y estudiantes en los primeros años de su implantación en los centros públicos de Andalucía. Málaga, Universidad de Málaga: Grupo de Investigación Nuevas Tecnologías aplicadas a la Educación.

Colas, P. y Casanova, J. (2010). Variables docentes y de centro que generan buenas prácticas con TIC. Revista Electrónica Teoría de la Educación: Educación y Cultura en la Sociedad de la Información. Vol. 11, 1, 121-147. 
Fandos, (2007). La telemática en los procesos educativos. Educans: una plataforma abierta para la educación secundaria obligatoria. Tesis doctoral. Universidad de Huelva.

Marchesi y Martin, (2003). Tecnología y aprendizaje. Investigación sobre el impacto del ordenador en el aula. Madrid: Editorial S.M.

Montero, L., Gewerc, A., Vidal Puga, M. P., Fernández Tilve, M. D., Rodríguez Rodríguez, J., González Guisande, O., González Fernández, R., Pernas, E. y Martínez Piñeiro, E. (2004). La Influencia de las nuevas tecnologías en el desarrollo organizativo y profesional de los centros educativos. Estudio de la situación en Galicia. Santiago de Compostela: Informe de investigación del Grupo Stellae (Inédito). Universidad de Santiago de Compostela.

Ortiz, A. (2006). La implantación de las TIC o el uso de Internet en la escuela. Innovación educativa, no 16, 31- 45.

Ortiz, A. (2005). Interacción y TIC en la enseñanza universitaria. Píxel-Bit, no 26, 27- 38. http://www.sav.us.es/pixelbit/pixelbit/articulos/n26/n26art/art2603.htm

Pérez, A, I. y Sola, M. (2006). La emergencia de buenas prácticas. Informe final. Evaluación externa de los proyectos educativos de centros para la incorporación de las nuevas tecnologías de la información y la comunicación a la práctica docente. Edita: Dirección General de Innovación Educativa y Formación del Profesorado. Junta de Andalucía.

Pérez, M.A.; Aguaded, J.I. y Fandos, M. (2010) Percepciones, retos y demandas de los directores y coordinadores de los centros TIC andaluces. Educatio Siglo XXI. 28(1), 259-316.

Valverde, J., Garrido, M.C. y Sosa, M.J. (2010). Políticas educativas para la integración de las TIC en Extremadura y sus efectos sobre la innovación didáctica y el proceso de enseñanza/aprendizaje: la percepción del profesorado. Revista de educación. 352, 99-124.

\section{Para citar este artículo:}

ORTIZ, A.M., PEÑAHERRERA, M. \& ORTEGA, J.M. (2012). Percepciones de profesores y estudiantes sobre las tic. Un estudio de caso. EDUTEC, Revista Electrónica de Tecnología Educativa, 41. Recuperado el dd/mm/aa de http://edutec.rediris.es/Revelec2/Revelec41/precepciones profesores estudiantes TIC.html 